\title{
Utilidad diagnóstica de un Cuestionario de Sueño y de la Escala de Somnolencia de Epworth en el Síndrome de Apneas/Hipopneas Obstructivas del Sueño (SAHOS) The usefulness of a Sleep Questionnaire and the Epworth Sleep Scale in the diagnosis of Obstructive Sleep Apnea Syndrome (OSAS) \\ José Luis Castillo C. ${ }^{1,2}$, Fernando Araya D. ${ }^{3}$, Gonzalo Bustamante F. ${ }^{2}$, Lorena Montecino R. ${ }^{2}$, Catalina Torres M. ${ }^{2}$, Sabrina Oporto S. ${ }^{2}$, Luis Segovia S. ${ }^{1}$ y Pablo Reyes S. ${ }^{2}$
}

Introduction: Obstructive sleep apnea syndrome (OSAS) is a common disease associated with significant morbidity, including excessive daytime sleepiness, cardiovascular disease and stroke. Method: We studied prospectively the patients sent to our sleep laboratory for polysomnography (PSG) during 6 months. One-hundred patients were interviewed with a sleep questionnaire, 3 of them were ruled out because the lacking of PSG and 2 who no completed the Epworth sleep scale (ESS). Results: Out of the 95 patients, there were 85 men and 10 women, with a mean age of $47.4 \pm 12.5$ years, obesity was found in $42.5 \%$, an ESS greater than 11 points was found in $56.8 \%$, loud snoring in $93.7 \%$, breathing cessation in $68.4 \%$, excessive daytime somnolence in 57.9\%. PSG revealed no OSAS in $14.7 \%$, slight OSAS in $27.4 \%$, moderate OSAS in $21 \%$ y severe OSAS in $36.8 \%$. Body mass index and breathing cessation reported by the couple had the highest discriminative power with a sensibility of $87 \%$ and specificity of $50 \%$ for ruled out severe OSAS. Conclusion: A severe OSAS is less probable when there is absence of breathing cessation during sleep reported by the couple and an IMC $<30 \mathrm{Kg} / \mathrm{m}^{2}$.

Key words: Epworth sleepiness scale, polysomnography, daytime sleepiness, sleep apneas, sleep questionnaire.

Rev Chil Neuro-Psiquiat 2009; 47 (3): 215-221

Recibido: 10 de junio de 2009

Aceptado: 14 de septiembre de 2009

No se registran conflictos de interés.

Clínica Santa María, Laboratorio de Sueño.

Departamento de Ciencias Neurológicas, Universidad de Chile.

Servicio de Neurología, Hospital del Salvador. 


\section{Introducción}

$\mathrm{E}$ 1 síndrome de apneas/hipopneas del sueño (SAHOS) presenta una alta prevalencia en la población adulta ${ }^{1-3}$, con importante morbilidad cardiovascular ${ }^{4,5}$ y cerebrovascular ${ }^{6,7}$, además de una mayor incidencia de accidentes automovilísticos $^{8,9}$. Existe un alto número de pacientes con SAHOS que no han sido diagnosticados debido a lo complejo y caro del estudio polisomnográfico (PSG) considerado el "gold standard" para su diagnóstico ${ }^{10}$.

Es importante considerar que el tratamiento con CPAP de los pacientes con SAHOS permite mejorar los síntomas y reducir los riesgos cardio y cerebrovascular, además de los costos relacionados con estas condiciones. Sería muy útil por lo tanto, disponer de un instrumento de tamizaje que permita detectar en diferentes poblaciones a los pacientes con SAHOS que requieren ser estudiados con PSG para optimizar su manejo.

Se han intentado diversos métodos con este objetivo, entre los que destacan varios cuestionarios y modelos clínicos de tamizaje ${ }^{11-18}$ y dispositivos portátiles ${ }^{19,20}$ con resultados muy variables y controvertidos.

En un trabajo recientemente publicado nuestro grupo detectó una alta frecuencia de trastorno de sueño no diagnosticados en pacientes de un centro de salud familiar ${ }^{21}$.

El objetivo de este estudio es conocer las características clínicas y polisomnográficas de los pacientes con sospecha de SAHOS en nuestro medio y la utilidad diagnóstica de un cuestionario de sueño y de la Escala de somnolencia de Epworth (EE).

\section{Método}

Durante un período de 6 meses, se estudió prospectivamente a los pacientes enviados para PSG al Laboratorio de Sueño de la Clínica Santa María, con sospecha clínica de SAHOS y solicitud de eventual titulación de CPAP.

Previo consentimiento informado, se entrevis- tó a cada sujeto con un cuestionario estructurado de sueño que incluye: antecedentes de enfermedades médicas, uso de medicamentos, tabaco y alcohol, hábitos de sueño, ronquido intenso, pausas respiratorias, insomnio, somnolencia diurna excesiva y la autoaplicada Escala de Somnolencia de Epworth para detectar somnolencia diurna excesiva ${ }^{22,23}$. Además, se midieron algunas variables antropométricas como peso, talla, presión arterial, circunferencia abdominal y cuello y se calculó el índice de masa corporal (IMC).

El estudio polisomnográfico incluyó: 6 canales de EEG; electro-oculograma (EOG); EMG en el mentón y en ambos tibiales anteriores; control de la respiración con thermocupla oronasal, PTAF (pressure transducer airflow) y esfuerzo toraco-abdominal; saturación de oxígeno; medición de ronquido; control de posición corporal y ECG. El análisis de la PSG fue realizada por uno de los autores (JLC), en ciego a los resultados del cuestionario y a la evaluación clínica de los pacientes.

Los pacientes se clasificaron como normales si tenían 5 o menos eventos respiratorios por hora (IAH: índice de apneas/hipopneas) y 90\% o más de saturación mínima de oxígeno; SAHOS leves: 6-14 IAH y 81-90\% de saturación mínima de oxígeno; moderados: 15-30 IAH y oximetría mínima de 70-80\% y SAHOS severo con más de 30 IAH y oximetría mínima de menos de $70 \%$.

A los pacientes que presentaron 15 o más IAH en la primera mitad de la noche, se les instaló CPAP, con aumentos progresivos de presión, hasta lograr la mayor disminución posible de las apneas/hipopneas durante el sueño. Esta es la modalidad de PSG denominada "noche dividida"24.

Análisis de los datos: El análisis estadístico fue llevado a cabo usando el programa SPSS para windows (versión 15). Las variables contínuas se presentan como promedio \pm DE y ellas se compararon usando ANOVA. Los datos categoriales se presentan como frecuencias y se compararon a través de chi cuadrado de Pearson. Mediante un análisis por regresión logística se seleccionaron aquellas variables que aportaron significativa- 
mente a la varianza del número de apneas/ hipopneas. Con ellas se confeccionó un modelo de predicción para clasificar un grupo de pacientes con un número de apneas/hipopneas $\leq 30$ por hora (SAHOS no severo), y otro grupo con un número $>30$ por hora (SAHOS severo). Se utilizó un valor de significancia $<0,05$, IC $=95 \%$ y poder de $80 \%$.

\section{Resultados}

Entre los meses de septiembre del 2007 y febrero del 2008, se estudiaron 95 pacientes, que completaron toda la evaluación requerida, con una edad promedio de 47,4 años $( \pm 12,5), 10$ mujeres y 85 hombres. La PSG dividió a los pacientes en 4 grupos de acuerdo a los criterios de severidad mencionados en el método. En la Tabla 1 se presentan las características de los grupos, des- tacando la mayor frecuencia de IMC sobre $30 \mathrm{~kg} /$ $\mathrm{m}^{2}$ en SAHOS severo $(\mathrm{p}<0,05)$. La Tabla 2 presenta los resultados de las variables clínicas del sueño, destacando la significativa menor frecuencia de reportes de observación de pausas en los pacientes sin SAHOS $(\mathrm{p}<0,05)$. En la Tabla 3, al analizar las características del hipnograma en la PSG, se encuentra que los pacientes sin SAHOS tienen un sueño más eficiente, con menos despertares y con más etapa REM, pero sin alcanzar en esta muestra significancia estadística.

Se separaron los pacientes en 2 grupos, con $\mathrm{IAH} \leq$ de 30 y $>30 /$ hora. Indice mayor de 30 corresponde a los pacientes con SAHOS severa y que por lo tanto requieren tratamiento.

Mediante un análisis de regresión logística, el IMC y las pausas respiratorias reportadas por la pareja, se identificaron como variables predictivas relevantes. Lo valores de los coeficientes de cada una fueron $1,15(\mathrm{p}=0,01$; IC $95 \%=1,03-1,3)$

Tabla 1. Características físicas principales

\begin{tabular}{lcccc}
\hline & No & Leve & SAHOS & Moderado \\
\hline $\mathrm{n}$ & 14 & 26 & 20 & Severo \\
Edad (años) & $40,4 \pm 12$ & $46,8 \pm 12,1$ & $56,89 \pm 10$ & $47,57 \pm 13,3$ \\
Cuello $(\mathrm{cm})$ & $40,4 \pm 4,2$ & $41,3 \pm 3,4$ & $41,2 \pm 4$ & $43,7 \pm 3,4$ \\
Cintura abdominal $(\mathrm{cm})$ & $97,1 \pm 12,6$ & $102,4 \pm 9,8$ & $102,8 \pm 10,5$ & $105,8 \pm 11$ \\
$\mathrm{IMC}\left(\mathrm{Kg} / \mathrm{m}^{2} \pm \mathrm{DE}\right)$ & $27,8 \pm 4,5$ & $28,9 \pm 4,5$ & $29 \pm 3,8$ & $31,9 \pm 4,3$ \\
$\mathrm{IMC} \geq 30^{*}$ & $4(28,6 \%)$ & $10(38,5 \%)$ & $9(45 \%)$ & $23(66 \%)$ \\
\hline
\end{tabular}

${ }^{\star} \mathrm{p}<0,05$

Tabla 2. Variables clínicas del sueño y Escala de Epworth (EE)

\begin{tabular}{|c|c|c|c|c|}
\hline & \multicolumn{4}{|c|}{ SAHOS } \\
\hline & No & Leve & Moderado & Severo \\
\hline Ronquido observadas (\%) & 92,3 & 92,3 & 90 & 97 \\
\hline Pausas observadas $(\%)^{\star}$ & 35,7 & 73 & 70 & 77,1 \\
\hline Somnolencia diurna (\%) & 78,6 & 57,7 & 65 & 45,7 \\
\hline Cansancio al despertar (\%) & 92,9 & 77 & 60 & 91,4 \\
\hline $\mathrm{EE}$ (puntaje promedio $\pm \mathrm{DE}$ ) & $10,7 \pm 3,6$ & $11.6 \pm 5,8$ & $12,6 \pm 4,9$ & $11,6 \pm 4,5$ \\
\hline
\end{tabular}

${ }^{*} \mathrm{p}<0,05$ 
Tabla 3. Características del hipnograma

\begin{tabular}{lcccc}
\hline & No & Leve & SAHOS & Moderado \\
\hline $\mathrm{n}$ & 14 & 26 & 20 & Severo \\
Eficiencia sueño (\%) & $81,5 \pm 8,5$ & $72,5 \pm 21,9$ & $73,1 \pm 14,3$ & $71,0 \pm 19,9$ \\
Despertares/hora & $6,3 \pm 6,7$ & $7,8 \pm 4,2$ & $10,1 \pm 6,6$ & $26,8 \pm 17,3$ \\
Etapa I (\%) & $20,8 \pm 8$ & $21,9 \pm 19,9$ & $23,7 \pm 10,6$ & $32,7 \pm 19,6$ \\
Etapa II (\%) & $51,6 \pm 9,3$ & $44,7 \pm 14,9$ & $49,2 \pm 11,3$ & $48,8 \pm 16,6$ \\
Etapa III/IV (\%) & $13,4 \pm 9,9$ & $20,6 \pm 14,9$ & $14,7 \pm 8,9$ & $9,0 \pm 8,3$ \\
Etapa REM (\%) & $13,0 \pm 6,3$ & $11,1 \pm 7,7$ & $11,9 \pm 6,7$ & $8,1 \pm 6,4$
\end{tabular}

para el IMC y $0,23(\mathrm{p}=0,016$; IC $95 \%=0,07$ $0,76)$ para las pausas. De este análisis se obtuvo un modelo confiable $\left(\chi^{2}=17,08, \mathrm{gl}=2, \mathrm{p}<0,001\right)$, que da cuenta de entre el $17 \%$ y $23 \%$ de la varianza del número de eventos respiratorios $\leq 30$. Un IAH $\leq 30$ fue predicho en forma exitosa en hasta el $87 \%$ de los pacientes, pero sólo el 50\% de las veces la predicción de un IAH $>30$ fue realizada con éxito. En total, el modelo predice exitosamente el $74 \%$ de los pacientes.

\section{Discusión}

La edad media y el IMC elevado de los pacientes estudiados, corresponde a lo habitualmente descrito en una población con alta sospecha clínica de SAHOS ${ }^{2,3}$. Llama la atención el escaso número de mujeres, que si bien presentan menos frecuentemente este síndrome, en la literatura internacional ocurre con una relación hombre mujer más estrecha ${ }^{2,3}$. Es posible que el subdiagnóstico del SAHOS, debido al alto costo de la PSG sea mayor en las pacientes mujeres, ya que tradicionalmente se tiende a sospechar más en hombres obesos y roncadores.

A partir de los principales síntomas y características físicas que presentan los pacientes con SAHOS, se han implementado varios cuestionarios y escalas que establecen grados de sospecha de este síndrome, pero la asignación a un grupo de alta probabilidad no ha podido ser hecha con suficiente seguridad como para eliminar la necesidad de la PSG ${ }^{16-18}$.

El cuestionario de sueño utilizado en este estudio reveló una utilidad limitada como instrumento de tamizaje de SAHOS que requiere CPAP. Aunque su capacidad de predicción de los pacientes con SAHOS no severo es bastante alta (87\%), no sucede lo mismo con los severos en que su predicción es prácticamente al azar (50\%).

Las dos variables más significativas en nuestro estudio (IMC y pausas respiratorias observadas) se encuentran también en la mayoría de los trabajos que intentan la detección de SAHOS ${ }^{11-18}$. El ronquido intenso fue descrito por la pareja y/o el paciente en la inmensa mayoría de nuestra muestra y en todos los grupos y probablemente fue uno de los factores considerados por los médicos tratantes para sospechar SAHOS y enviarlos al examen de PSG, lo que explicaría que en este estudio no tenga capacidad discriminativa.

A diferencia del trabajo de Maislin ${ }^{12}$, no encontramos que las variables sexo y edad contribuyeran a aumentar la sospecha de SAHOS. Es posible que la mayoritaria presencia de hombres en nuestra muestra impida que el factor género discrimine en la probabilidad de tener o no SAHOS.

La Asociación Americana de Trastornos de Sueño plantea la estratificación de los pacientes, en base a la presencia de los siguientes factores: ronquido habitual, somnolencia diurna excesiva, IMC > 35 y observación de pausas respirato- 
rias nocturnas ${ }^{25}$. Los pacientes con estos 4 factores tendrían una alta probabilidad de tener apneas de sueño y pueden ser clasificados como grupo de alto riesgo $(70 \%$ de probabilidades de tener un índice de apneas/hipopneas de al menos 10 por hora). La severidad de estos factores de riesgo se correlaciona a menudo con la gravedad del SAHOS, sin embargo, la mayoría de los estudios revela un valor predictivo limitado ${ }^{25}$.

El Cuestionario de Berlín es uno de los instrumentos de tamizaje más usados ${ }^{13}$. Su rendimiento predictivo es variable dependiendo de las diferentes poblaciones de pacientes estudiados. En los pacientes referidos a un laboratorio de sueño revela un muy bajo valor predictivo, con sensibilidad y especificidad de 68 y $49 \%$ para índices de apneas/hipopneas mayores de 5 y de 57 y $43 \%$ para índices mayores de $15^{18}$. Además, ha sido validado usando dispositivos portátiles que no son aceptados como el estándar para el diagnóstico de SAHOS $^{20}$.

El "apnea risk evaluation system" (ARES) que combina elementos del cuestionario de Berlin, el índice de Flemons y la escala de Epworth, tiene una sensibilidad de $94 \%$ y una especificidad de $79 \%{ }^{15}$.

El año 2006, la Sociedad Americana de Anestesiología publicó guías para el manejo de los pacientes con SAHOS, debido a que es un factor de riesgo importante para el desarrollo de eventos adversos perioperatorios ${ }^{26}$. Un grupo de anestesistas creó el cuestionario STOP (snoring, tiredness during daytime, observed apneas and high blood pressure) para el tamizaje de SAHOS ${ }^{16}$. Validado en 2.467 pacientes quirúrgicos, se encontró que tenía una sensibilidad de 79,5\% para índices de apneas/hipopneas mayores de $30 \mathrm{y}$ al incorporar el IMC, edad, circunferencia de cuello y género la sensibilidad alcanzaba más del $90 \%$ para SAHOS moderados y severos, con un alto valor predictivo positivo para SAHOS en pacientes hombres, mayores de 50 años, con IMC mayor de $35 \mathrm{~kg} / \mathrm{m}^{2}$ y circunferencia de cuello mayor de $40 \mathrm{~cm}$. Un estudio muy reciente prospectivo observacional en 2.877 pacientes quirúrgicos, utilizó el ARES, encontrando en $25 \%$ con alto riesgo para SAHOS que fue detectado posteriormente con dispositivos ambulatorios ${ }^{27}$.

La escala de de Epworth es uno de los instrumentos más utilizados en la detección de somnolencia y por tratarse de uno de los principales síntomas del SAHOS se ha intentado su uso como método de tamizaje. Actualmente, un puntaje de 10 o más se considera como el punto de corte más apropiado para detectar somnolencia pato$\operatorname{lógica}^{28}$. En nuestro estudio la escala de somnolencia de Epworth se correlacionó pobremente con el número de apneas registradas en la PSG. De esta manera, la escala de Epworth en nuestra muestra no contribuye a la detección de pacientes con SAHOS a pesar de que la somnolencia es un síntoma cardinal de este síndrome, lo que está de acuerdo con resultados similares de un estudio reciente que reveló una escasa capacidad discriminativa de esta escala para el tamizaje del SAHOS $^{29}$.

Las variables más relevantes encontradas en nuestro estudio son el IMC y la observación por parte de la pareja, de pausas en la respiración durante el sueño. Por lo tanto, recomendamos su inclusión en la evaluación de los pacientes con sospecha de SAHOS. 


\section{Resumen}

Introducción: El SAHOS presenta una alta prevalencia en estudios internacionales, con importantes repercusiones en los sistemas cardio, cerebrovascular y en la calidad de vida de las personas. Método: Estudiamos prospectivamente a los pacientes enviados para Polisomnografia (PSG) a nuestro laboratorio de sueño, en un período de 6 meses. Previo consentimiento informado, se les aplicó la Escala de Somnolencia de Epworth (EE) y un Cuestionario de Sueño. Fueron entrevistados 100 pacientes, excluyéndose 3 por no haberse realizado PSG y 2 que no completaron la EE. Resultados: Se analizaron 95 pacientes, 89,5\% hombres, edad promedio 47,4 12,5 años, obesidad en 49,5\%, EE mayor de 11 en 56,8\%, ronquido en 93,7\%, pausas respiratorias en $68,4 \%$, somnolencia diurna excesiva en $57,9 \%$, cansancio al despertar en 86,3\%. La PSG no demostró SAHOS en 14,7\%, hubo 27,4\% con SAHOS leve, $21 \%$ moderado y 36,8\% severo. Las variables presencia de pausas respiratorias observadas por la pareja e indice de masa corporal (IMC) predicen la ausencia de SAHOS severo, con una sensibilidad de $87 \%$ y especificidad de 50\%. Conclusión: En la evaluación de pacientes con sospecha de SAHOS, la no observación de pausas respiratorias durante el sueño por parte de la pareja y el IMC menor de $30 \mathrm{Kg} / \mathrm{m}^{2}$ hacen menos probable que exista un SAHOS severo.

Palabras clave: Apneas de sueño, cuestionario de sueño, polisomnografía, escala de somnolencia de Epworth, somnolencia diurna excesiva.

\section{Referencias}

1. Leger D, Poursain B, Neubauer D, Uchiyama M. An international survey of sleeping problems in the general population. Curr Med Res Opin 2008; 24 (1): 307-17.

2. Young T, Evans L, Finn L, Palta M. Estimation of the clinically diagnosed proportion of sleep apnea syndrome in middle-aged men and women. Sleep 1997; 20: 705-6.

3. Young T, Peppard P E, Gottlieb D J. Epidemiology of obstructive sleep apnea: a population health perspective. Am J Respir Crit Care Med 2002; 165: 1217-39.

4. Shamsuzzaman A S M, Gersh B J, Somers V K. Obstructive sleep apnea. Implications for cardiac and vascular disease. JAMA 2003; 290: 1906-14.

5. Quan S F, Gersh B J. Cardiovascular consequences of sleep disordered breathing: past, presente and future. Circulation 2004; 109: 951-7.

6. Bassetti C L, Milanova M, Gugger M. Sleep disordered breathing and acute ischemic stroke. Stroke 2006; 37: 967-72.
7. Yaggi H K, Concato J, Kernan W N, Lichtmann J H, Brass L M, Moshsenin V. Obstructive sleep apnea as a risk factor for stroke and death. $\mathrm{N}$ Engl J Med 2005; 353: 2034-41.

8. Turkington P M, Sircar M, Allgar V, Elliott M W. Relationship between obstructive sleep apnoea, driving simulator performance and risk of road traffic accidents. Thorax 2001; 56: 800-5.

9. George C F P. Sleep 5: Driving and automobile crashes in patients with obstructive sleep apnoea/ hypopnoea syndrome. Thorax 2004; 59: 804-7.

10. Young T, Evans L, Finn L, Palta M. Estimation of the clinically diagnosed proportion of sleep apnea syndrome in middle-aged men and women. Sleep 1997; 20 (9): 705-6.

11. Douglass A B, Bornstein R, Nino-Murcia G, Keenan $\mathrm{S}$, Miles L, Zarcone V P, et al. The sleep disorders questionnaire I: Creation and multivariate structure of SQD. Sleep 1994; 17 (2): 160-7.

12. Maislin G, Pack AI, Kribbs N B, Smith P L, Schwartz A R, Kline L R, et al. A survey screen for prediction of apnea. Sleep 1995; 18: 158-66

13. Netzer N C, Stoohs R A, Netzer C M, Clark K, 
Strohl KP. Using the Berlin Questionnaire to identify patients at risk for the sleep apnea syndrome. Ann Intern Med 1999; 131: 485-91.

14. Weatherwax K J, Lin X, Marzec M L, Malow B A. Obstructive sleep apnea in epilepsy: the Sleep Apnea scale of the Sleep Disorders Questionnaire (SASDQ) is a useful screening instrument for obstructive sleep apnea in a disease-specific population. Sleep Medicine 2003; 4: 517-21.

15. Levendowski D J, Olmstead R, Popovic D, Carper D, Berka C, Westbrook PR. Validation of an apnea risk evaluation questionnaire. Sleep Diagn Ther 2007; 2 (2): 20-6.

16. Chung F, Yegneswaran B, Liao P, Chung S A, Vairavanathan S, Islam S, et al. STOP Questionnaire. A tool to screen patients for obstructive sleep apnea. Anesthesiology 2008; 108: 812-21.

17. Chung F, Yegneswaran B, Liao P, Chung SA, Vairavanathan S, Islam S, et al. Validation of the Berlin Questionnaire and American Society of anesthesiologist checklist as screening tools for obstructive sleep apnea in surgical patients. Anesthesiology 2008; 108: 822-30.

18. Ahnmadi N, Chung S A, Gibbs A, Shapiro C M. The Berlin Questionnaire for sleep apnea in a sleep clinic population: Relationship to polysomnographic measurements of respiratory disturbance. Sleep Breath 2008; 12: 39-45.

19. Parks P D, Durand G, Tsismenakis A J, Vela-Bueno A, Kales S N. Screening for obstructive sleep apnea during commercial driver medical examinations. J Occup Environ Med 2009; 51: 27582.

20. Task force members: Collop N A, McDonwell Anderson W, Boehlecke B, Claman D, Goldberg R, Gottlieb DJ, Hudgel D, Sateia M, Schwab R. Clinical guidelines for the use of unattended portable monitors in the diagnosis of obstructive sleep apnea in adult patients. J Clin Sleep Med 2007; 3 (7): 73747.

21. Castillo J L, Araya F, Montecino L, Torres C, Oporto S, Bustamante G, et al. Aplicación de un cuestionario de sueño y la escala de somnolencia de Epworth en un centro de salud familiar. Rev Chil Neuro-Psiquiat 2008; 46: 191-200.

22. Johns M W. A new method for measuring daytime sleepiness. Sleep 1991; 14: 540-5.

23. Chiner E, Arriero J M, Signes-Costa J, Marco J, Fuentes I. Validación de la versión española del test de somnolencia de Epworth en pacientes con síndrome de apnea de sueño. Arch Bronconeumol 1999; 35: 422-7.

24. Kushida C A, Chediak A, Berry R B, Brown L K, Gozal D, Iber C, et al. Clinical guidelines for the manual titration of positive airway pressure in patients with obstructive sleep apnea. J Clin Sleep Med 2008; 4: 157-71.

25. Kushida C A, Littner M R, Morgenthaler T, Aessi C A, Bailey D, Coleman J Jr, et al. Practice parameters for the indications for polysomnography and related procedures: an update for 2005. Sleep 2005; 28: 499-521.

26. Gross J B, Bachenberg K L, Benumof J L, Caplan R A, Conis R T, Cote C J, et al. American Society of Anesthesiologists Task Force on Perioperative Management. Practice guidelines for the perioperative management of patients with obstructive sleep apnea: a report by the American Society of Anesthesiologists Task Force on Perioperative Management of patients with obstructive sleep apnea. Anesthesiology 2006; 104 (5): 1081-93.

27. Finkel K J, Searleman A C, Tymkew H, Tanaka Ch Y, Saager L, Safer-Zadeh E, et al. Prevalence of undiagnosed obstructive sleep apnea among adult surgical patients in an academic medical center. Sleep Medicine 2009, doi:10.1016/j.sleep.2008.08.007.

28. Johns M W. Sensitivity and specificity of the multiple sleep latency test (MSLT), the maintenance of wakefulness test and the Epworth sleepiness scale: failure of the MSLT as a gold standard. J Sleep Res 2000; 9 (1): 5-11.

29. Rosenthal L D, Colan D C. The Epworth sleepiness scale in the identification of obstructive sleep apnea. J Nerv Ment Dis 2008; 196: 429-31.

Correspondencia:

José Luis Castillo C.

JM Infante 553. Providencia. Santiago. Chile

Fono-fax: 562-2360170

E-mail: jcastill@med.uchile.cl 